INPLASY

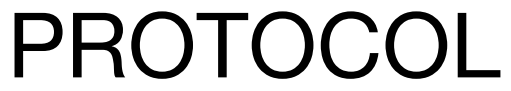

To cite: $\mathrm{Ba}$ et al. The Predictive Role of Circulating Tumor DNA in Melanoma Patients Treated with Immune Checkpoint Inhibitors: A Systematic Review and Meta-Analysis. Inplasy protocol 202110114. doi:

10.37766/inplasy2021.1.0114

Received: 28 January 2021

Published: 29 January 2021

Corresponding author:

$\mathrm{He} \mathrm{Ba}$

bahe2474364@163.com

Author Affiliation:

The First Affiliated Hospital of Anhui Medical University, Hefei 230022

Support: NSFC (81693439).

Review Stage at time of this submission: Preliminary searches.

Conflicts of interest: None.

\section{The Predictive Role of Circulating Tumor DNA in Melanoma Patients Treated with Immune Checkpoint Inhibitors: A Systematic Review and Meta-Analysis}

$\mathrm{Ba}, \mathrm{H}^{1}$; Chen, J2; Zhu, YD².

Review question / Objective: What is the association between circulating tumor DNA(ctDNA) level and prognosis of melanoma patients receiving immune checkpoint inhibitors(ICls) therapy.

Condition being studied: Immune checkpoint inhibitors (ICls) treatment has effectively improved response and survival of melanoma patients. However, ICls is not always effective in all TMB (tumor mutation burden) high or PD-L1 positive patients. Therefore, the need to identify reliable biomarkers in response predicting is still urgent. Circulating tumor DNA (ctDNA), with the advantage of easy to obtain and noninvasive, has been shown to be a promising biomarker in melanoma. Increasing studies had reported ctDNA as an early predictor of tumor response and clinical benefit. In this meta-analysis, we aimed to ascertain the predictive value of ctDNA levels at baseline and during treatment in melanoma patients received ICls therapy.

INPLASY registration number: This protocol was registered with the International Platform of Registered Systematic Review and Meta-Analysis Protocols (INPLASY) on 29 January 2021 and was last updated on 29 January 2021 (registration number INPLASY202110114).

\section{INTRODUCTION}

Review question / Objective: What is the association between circulating tumor DNA(ctDNA) level and prognosis of melanoma patients receiving immune checkpoint inhibitors(ICls) therapy.
Condition being studied: Immune checkpoint inhibitors (ICls) treatment has effectively improved response and survival of melanoma patients. However, ICls is not always effective in all TMB (tumor mutation burden) high or PD-L1 positive patients. Therefore, the need to identify reliable biomarkers in response predicting is still 
urgent. Circulating tumor DNA (ctDNA), with the advantage of easy to obtain and noninvasive, has been shown to be a promising biomarker in melanoma. Increasing studies had reported ctDNA as an early predictor of tumor response and clinical benefit. In this meta-analysis, we aimed to ascertain the predictive value of ctDNA levels at baseline and during treatment in melanoma patients received ICls therapy

\section{METHODS}

Search strategy: PubMed, Embase, Cochrane, Web of Science and ClinicalTrials.gov will be searched from inception to 03 March, 2021 for studies published in English by two investigators independently. The search terms are as follows: (PD-1 OR PD-L1 OR CTLA4 OR Ipilimumab OR Nivolumab OR Tremelimumab OR Pembrolizumab OR Avelumab OR Atezolizumab OR Durvalumab OR "immune checkpoint inhibitor" OR "immune checkpoint inhibitors" OR "ICI" OR "ICls" OR "immune checkpoint blocker" OR "immune checkpoint blockers" OR "ICB" OR "ICBs") AND (cell free DNA OR circulating tumor DNA or circulating DNA OR Liquid Biopsy OR cfDNA OR ctDNA OR extracellular DNA) AND melanoma.

Participant or population: Melanoma patients treated with inhibitors of PD-1/PDL1, CTLA-4, or their combination, or combined with chemotherapy.

Intervention: Patients with undetectable ctDNA before ICIs treatment or with decreased ctDNA concentration during treatment

Comparator: Patients with detectable ctDNA before ICIs treatment or with increased ctDNA concentration during treatment.

Study designs to be included: Clinical trials or cohort studies.

Eligibility criteria: (1) Clinical trials or cohort studies assessed inhibitors of PD-1/PD-L1,
CTLA-4, or their combination, in patients with melanoma, the baseline evaluation of ctDNA was dichotomized as detectable or undetectable, or change of ctDNA level during treatment was recorded. (2) Hazard ratio (HR) of progression-free survival (PFS) or overall survival (OS), and their $95 \%$ confidence intervals $(95 \% \mathrm{Cl})$ were given in the article.

Information sources: PubMed, Embase, Cochrane, Web of Science and ClinicalTrials.gov .

Main outcome(s): Overall survival (OS), progression free survival (PFS).

Quality assessment / Risk of bias analysis: The Newcastle-Ottawa Scale (NOS) will be adopted to assess the quality of studies included. The total score ranged from 0 to 9, as 8-9 points indicates high quality of a study, five to seven points indicates medium quality, and points lower than five indicates poor quality.

Strategy of data synthesis: Heterogeneity among individual studies will be evaluated by the $Q$ test; $12>50 \%$ or $P<0.10$ indicates significant heterogeneity. Pooled HR with Z test will be calculated by random-effects model. Funnel plots will be constructed, and Begg's test and Egger's test will be performed to evaluate publication bias $(P<$ 0.10 will be considered as visible publication bias). Sensitivity analysis will be used to test the stability of the results in the meta-analysis.

Subgroup analysis: Subgroup analyses stratified by metastatic or locally of the melanoma will be conducted.

Sensitivity analysis: (1) Observe changes in the statistical result after studies with the largest and smallest weight are excluded. (2) Synthesis the data with random-effects model and fixed-effects model successively (3) Overall subgroup analyses will also be carried out to identify potential source of heterogeneity.

Country(ies) involved: China. 
Keywords: ctDNA, circulating tumor DNA, melanoma, immune checkpoint inhibitor, prognosis.

Contributions of each author:

Author 1 - He Ba - Literature searching and manuscript writing.

Email: bahe2474364@163.com

Author 2 - Jie Chen - Literature searching and data extracting.

Author 3 - Yaodong Zhu.

Email: zhuyaodong2013@163.com 